\title{
A New Comprehensive Framework for Ranking Accepted Orders and Supplier Selection in Make-To-Order Environments
}

\author{
Masoud Rabbani ${ }^{1}$, Ghazaleh Ahmadi ${ }^{2}$, Ramez Kian ${ }^{3}$ \\ ${ }^{1}$ Department of Industrial Engineering, Engineering College, Tehran University, Iran (mrabani@ut.ac.ir) \\ ${ }^{2}$ Department of Industrial Engineering, Engineering College, Tehran University, Iran (ahmadigh@ut.ac.ir) \\ ${ }^{3}$ Department of Industrial Engineering, Sharif University of Technology, Iran (ramez@ie.sharif.edu)
}

\begin{abstract}
This paper presents a new decision making framework for supplier selection in a make-to-order-system (MTO) while establishing an information loop between "sale" and "purchasing" functions which play important roles in successful satisfying of customers. The order entry stage outputs, including accepted orders' requirements and their priorities are reflected into the supplier selection phase; and in return, the outputs of the supplier selection model are fed back into the sale department to revise the orders attributes according to the system's supplying capabilities. A ranking procedure using Fuzzy data-envelopment-analysis (DEA) and multi attribute utility model (i.e. similarity to ideal solution TOPSIS) is applied to determine the relative ranking of accepted orders; in the next phase a goal programming supplier selection model focusing on three criteria of time, cost and quality is run to determine the best ability of the system in supplying required components and materials of the accepted orders.
\end{abstract}

Keywords: make-to-order system, supplier selection, order entry stage

\section{INTRODUCTION}

Today's market environment is characterized by diverse customer tastes and preferences, rapid developments in technology, and the management of globalization [1]. In such a situation using Make-to-stock (MTS) systems are risky and expensive as well. So build-to-order (BTO)/ make-to-order (MTO) systems have received a great deal of attention in recent years.

For a BTO/MTO manufacturing organization to have the benefit of competitive advantage in the business environment, it is important to make an integrated partnership relationship with its suppliers. So the supplier selection decision becomes crucial due to its significant effect on the successful building of an efficient supply chain. In an MTO system, the necessity of considering delivery date, quality and cost rather than setting cost as the only criterion of supplier evaluation becomes much clearer. On the other hand, due to the limitation of capacity and scarcity of resources, in an MTO system customers fall into different priority classes and it is important to link the order entry stage of the system with the procurement stage.

This paper tries to build a new comprehensive decision making structure for supplier selection considering the ranking of accepted orders. This structure is made up of two major parts. In the first part, a DEA and fuzzy multi attribute utility theory (i.e. technique for order preference by similarity to an ideal solution, TOPSIS) have been applied to rank the accepted orders; and in the second part a mixed integer goal programming considering goals of cost, quality and delivery time has been developed for supplier selection.

\section{LITERATURE REVIEW}

\subsection{Fuzzy Data Envelopment Analysis}

Traditional DEA models such as CCR models [2] require crisp input and output data. However it is not the case in most real world situations. Sengupta [3] proposed a fuzzy mathematical programming approach which incorporated fuzziness into a DEA model by defining tolerance levels on both objective function and constraint violations. Triantis and Girod [4] suggested a mathematical programming approach through transforming fuzzy input and output data into crisp ones using membership function values. Efficiency scores were computed for different values of membership functions and then averaged. Guo and Tanaka [5] proposed a fuzzy CCR model in which fuzzy constraints including fuzzy equalities and fuzzy inequalities were converted into crisp constraints by predefining a possibility level and using the comparison rule for fuzzy numbers. Wang et al. [6] propose two new fuzzy DEA models, which are constructed from the perspective of fuzzy arithmetic. They considered the most commonly used fuzzy numbers which are triangular and trapezoidal fuzzy numbers and formulated their new fuzzy DEA models as LP models without the need of making any assumptions. Solving the models will determine fuzzy efficiencies of DMUs. They used an analytical fuzzy ranking approach to compare and rank the efficiencies. 


\subsection{Fuzzy TOPSIS}

TOPSIS, first developed by Hwang and Yoon [7], is one of the known classical multi-attribute decision-making methods. In classical MCDM methods, the ratings and the weights of the criteria are known precisely [7]. However, in the real-world, these parameters may encounter with uncertainties. A more realistic approach may be to use linguistic assessments instead of numerical values. Therefore, fuzzy sets theory, introduced by Zadeh [8], was put into account to resolve the vagueness, ambiguity and subjectivity of human judgment.

Some studies have provided interesting results on TOPSIS with the help of fuzzy sets theory. For example Wang and Elhag [9] proposed a fuzzy TOPSIS method based on alpha level sets and suggested a nonlinear programming (NLP) solution procedure.

\subsection{Supplier Selection}

Ghodsypour and O'Brien [10] developed a mixed integer non-linear programming to solve the multiple sourcing problems, which takes into account the total cost of logistics, including net price, storage, transportation and ordering costs. Buyer limitations such as budget, quality and service were also considered in the model. They extended their single objective model to a multi-objective programming model to add different criteria. As mentioned by them, the idea of multiple objectives programming is to minimize the deviations from each goal, in order of priority. Higher priority goals were satisfied at the expense of lower priority goals. Cakravastia and Nakamura [11] developed a model for price and due date negotiations between a manufacture and its multiple suppliers to fulfill a single order from a customer. Liao and Kuhn [12] proposed a multi-objective program for a single item model under the assumption that all suppliers' lots simultaneously arrive at the beginning of each replenishment period. The objectives are the minimization of the total cost, the total quality rejection and the total delivery late while the constraints concern the capacity and demand satisfaction. To deal with the multi-objective optimization, a Genetic Algorithm was applied.

Ebadian et al. [13] presented a comprehensive decision making structure for production planning in an MTO system to reject or accept the new arriving orders with respect to the system limitations such as capacity. The last step of this structure was the selection of the best set of suppliers and subcontractors to provide the materials and workload for the accepted orders. This set was determined with respect to the price and delivery date of the required materials quoted by the suppliers. Assuming the same quality level for all suppliers and subcontractors, as well as single sourcing for each supplying item, they developed a mixed integer programming model. Their objective function was to minimize the total cost of purchasing; lateness and earliness of received orders.

\section{PROBLEM DESCRIPTION}

Since in make-to-order companies, customers' orders are various and the capacity available to respond the orders on time is limited, MTO companies usually follow an order acceptance/rejection policy in their order entry stage to assure the compatibility of their capacities with the accepted orders. In this situation customers' orders fall into different priority classes according to the customers' attributes or their orders' features. These priorities should be taken into account in production planning stages and procurement stages as well to assure integration within the make-to-order supply chain. Since the supplier selection and order quantity allocation are the main tasks of procurement planning and they consist a significant portion of cost an delivery time of fulfilling customer orders, the customers' orders ranking should be passed on to supplier selection stage as input parameters.

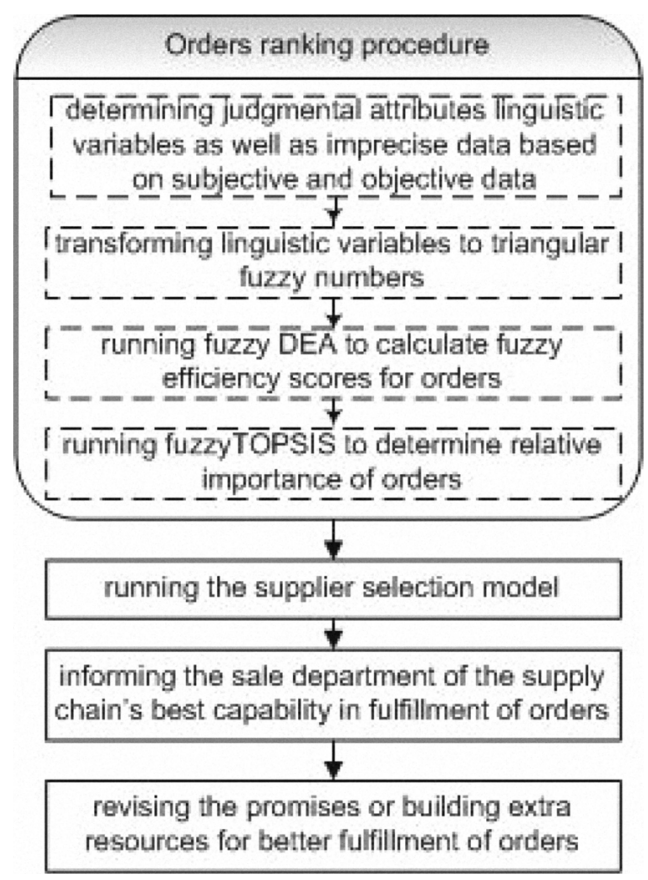

Figure1. Proposed decision making structure

In this paper, a comprehensive decision making structure is proposed for this purpose. The main idea of this structure is to present a model for supplier selection and allocating supplying quantities to the selected suppliers in a way that customers' requirement and order entry stage's outputs are projected. To do so, a goal programming approach is chosen to compare the desirability of different possible solutions with each other. Key success factors of quality, cost, and delivery time as well as the company utility values for accepted orders are the focusing parameters. The figure.1 
illustrates our proposed decision making structure. Quality, cost and delivery time are set according to the initial negotiation process between the company and the customers. To determine the company's utility values for orders, a ranking procedure is applied.

\section{DECISION MAKING STRUCTURE}

\subsection{Order Ranking Procedure}

A set of attributes including two particular categories of customer-related and order-related attributes are used in the order ranking procedure. Customer loyalty, customer credit, their access to the information technology, and their flexibility in the negotiation are the attributes in the customer-related category. Alignment of the order with the company's strategies, the amount of coordination and customization effort, the required non-regular capacities, and the order's contribution to the company's profit and market share are included in the order-related category.

procedure, first a fuzzy DEA is applied to calculate an efficiency score for each accepted order based on required customization efforts and non-regular capacity as inputs and market share and profit as outputs. These attributes are almost related and it is almost impossible to determine the best level of each of these attributes without considering the others. The model used here to measure efficiency scores was adopted from Wang et al. [6] and is as follows. In this model, $\left(x_{i j}^{L}, x^{M}, x_{i j}^{U}\right)$ presents fuzzy input and $\left(y_{i j}^{L}, y^{M}, y_{i j}^{U_{i j}}\right)$ present fuzzy output. $u_{\mathrm{r}}$ and $\mathrm{v}_{\mathrm{i}}$ are output and input multipliers respectively. $\left(\theta_{j}^{l}, \theta_{j}^{m}, \theta_{j}^{u}\right)$, the outputs of three fuzzy DEA models present orders' efficiency scores and are inputs to the multi attribute utility model in the next step.

Each DEA submodel has got $(1+n+s)$ constraints and $(s+m)$ variables. A total of $(3 n)$ problems should be solved to compute fuzzy efficiencies. All models could be solved with commercial mathematical software like LINGO within reasonable time.

$$
\max \theta_{o}^{L}=\sum_{r=1}^{s} u_{r} y_{r o}^{L}
$$

Subject to

$$
\begin{aligned}
& \sum_{i=1}^{m} v_{i} x_{i 0}^{U}=1 \\
& \sum_{r=1}^{s} u_{r} y_{r j}^{U}-\sum_{i=1}^{m} v_{i} x_{i j}^{L} \leq 0 \quad j=1, . ., n \\
& u_{r}, v_{i} \geq 0 \quad i=1, . ., m ; \quad r=1, . ., s \\
& \max \theta_{o}^{M}=\sum_{r=1}^{s} u_{r} y_{r o}^{M}
\end{aligned}
$$

$$
\begin{aligned}
& \sum_{i=1}^{m} v_{i} x_{i o}^{M}=1 \\
& \sum_{r=1}^{s} u_{r} y_{r j}^{U}-\sum_{i=1}^{m} v_{i} x_{i j}^{L} \leq 0 \quad j=1, . ., n \\
& u_{r}, v_{i} \geq 0 \quad i=1, . ., m ; \quad r=1, . ., s \\
& \max \theta_{o}^{U}=\sum_{r=1}^{s} u_{r} y_{r o}^{U}
\end{aligned}
$$

Subject to

$$
\begin{aligned}
& \sum_{i=1}^{m} v_{i} x_{i o}^{L}=1 \\
& \sum_{r=1}^{s} u_{r} y_{r j}^{U}-\sum_{i=1}^{m} v_{i} x_{i j}^{L} \leq 0 \quad j=1, . ., n \\
& u_{r}, v_{i} \geq 0 \quad i=1, . ., m ; \quad r=1, . ., s
\end{aligned}
$$

TOPSIS is a modified form of multi attribute utility theory. Since the attributes are not deterministic and articulated in fuzzy logic, a fuzzy TOPSIS is used. Now judgmental attributes as well as efficiency scores are put in a fuzzy TOPSIS. Fuzzy TOPSIS model used here is a combination of models developed by Yang et al. [14] and Butler et al. [15]. Similar to the work of Butler, probability is not involved in our scenario, so each attribute has got one state which is stated in fuzzy numbers transformed from linguistic variables along with a fuzzy efficiency score. A shortfall of TOPSIS is it does not involve a method for weighting attributes. As suggested by Butler [15], a conjoint analysis can be applied to determine the weights. The remainder of the model is adopted from Yang et al.[14]. The model formulation is as follows.

$$
D=A_{i}\left[\begin{array}{ccccc}
X_{1} & & X_{2} & & X_{3} \\
u_{11} & \cdots & u_{1 j} & \cdots & u_{1 n} \\
\vdots & & & & \vdots \\
u_{i 1} & \cdots & u_{i j} & \cdots & u_{i n} \\
\vdots & & & & \vdots \\
u_{m 1} & \cdots & u_{m j} & \cdots & u_{m n}
\end{array}\right] \text { (13) }
$$

Uij represents fuzzy utility value of attribute $j$ of alternative $I$ defined by a triangular fuzzy number $\left(\widetilde{u}_{i j}^{l}, \widetilde{u}_{i j}^{m}, \widetilde{u}_{i j}^{u}\right)$. In the next step the utility matrix is normalized. A linear transformation is used to normalize utility values which are shown in the following equations.

$$
\widetilde{v}_{i j}=\frac{\tilde{u}_{i j}}{\tilde{u}_{j}^{+}}=\left(\frac{a_{i j}}{c_{j}^{+}}, \frac{b_{i j}}{b_{j}^{+}}, \frac{c_{i j}}{a_{j}^{+}}\right)
$$

Subject to 


$$
\tilde{v}_{i j}=\frac{\tilde{u}_{j}^{-}}{\widetilde{u}_{i j}}=\left(\frac{a_{j}^{-}}{c_{i j}}, \frac{b_{j}^{-}}{b_{i j}}, \frac{c_{j}^{-}}{a_{i j}}\right)
$$

When $X j$ is an increasingly ordering attribute, equation 14 is used and when $X j$ is a decreasingly ordering attribute, equation 15 is used. for a given attribute $j$, the value $\tilde{u}_{i j}$ which has the largest generalized mean and the relatively small spread is defined as $\tilde{u}_{j}^{+}$the value $\tilde{u}_{i j}$ which has the smallest mean and the relative large standard deviation is defined as $\tilde{u}_{j}^{-}$. After applying normalization equations a normalized decision matrix $V$ is obtained where the cells $v_{i j}$ contain the normalized attribute values for the $i t h$ order on the $j$ th attribute.

The difference between attribute values $v_{i j}$ can be measured using their individual distances to the best and worst values using $\mathrm{D}_{\mathrm{ij}}^{+}$and $\mathrm{D}_{\mathrm{ij}}^{-}$. The difference measures between two fuzzy numbers, $\widetilde{v}_{i j}(x)$ which is a given order's, attribute value, and $\widetilde{v}_{j}^{+}(x), \widetilde{v}_{j}^{-}(x)$ which are the best and the worst attribute values in the jth chosen attribute set, can be defined as (Rilett and Park [16]; Zimmermann [17]):

$$
\begin{aligned}
& \widetilde{D}_{i j}^{+}\left(\widetilde{v}_{i j}, \tilde{v}_{j}^{+}\right)=1-\left\{\sup _{x}\left[\widetilde{v}_{i j}(x) \wedge \widetilde{v}_{j}^{+}\right]\right\} \\
& \widetilde{D}_{i j}^{-}\left(\widetilde{v}_{i j}, \widetilde{v}_{j}^{-}\right)=1-\left\{\sup _{x}\left[\widetilde{v}_{i j}(x) \wedge \widetilde{v}_{j}^{-}\right]\right\}
\end{aligned}
$$

Separation measures of $\mathrm{s}+\mathrm{i}$ and $\mathrm{s}-\mathrm{i}$ are computed according to the equations 18 and 19 .

$$
\begin{aligned}
& S_{i}^{+}=\sum_{j=1}^{n} D_{i j}^{+} w_{j}^{*} \\
& S_{i}^{-}=\sum_{j=1}^{n} D_{i j}^{-} w_{j}^{*}
\end{aligned}
$$

In order to rank the order, a relative closeness index based on the separation measures is developed as follows. This closeness index could be simply changed into orders relative weights.

$$
C_{i}=\frac{S_{i}^{-}}{S_{i}^{+}+S_{i}^{-}}
$$

\subsection{Supplier Selection Model}

\section{Indices:}

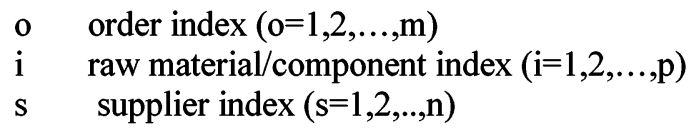

\section{Parameters:}

$M \quad$ a large number

$P T_{i s}$ production time of each unit of $i$ by supplier $s$

$D T_{s}$ transportation time from supplier $s$ to the company

$E T_{o}$ engineering time for design and customization of order $o$

$Q_{i s} \quad$ quality score of supplier $s$ for production of item $i$

$P C_{i s}$ production cost of each unit of $i$ by supplier $s$

$O C_{i s}$ cost of outsourcing each unit of the workload of $i$ by supplier $s$

$D C_{i s}$ cost of transportation for each unit of item $i$ from supplier $s$ to MTO company

$E C_{o}$ cost of design and engineering activities for order

$C_{s} \quad$ maximum available capacity of supplier $s$

$L_{s} \quad$ The minimum acceptable batch size for supplier $s$

$T G_{o}, C G_{o}, Q G_{o}$ Ideal completion time, cost and quality for order $o$

$D_{o}$ end product demand in order $o$

$R_{i o} \quad$ consumption rate of $i$ in $o$ respectively

$a_{O T}, a_{O C}, a_{O Q}$ importance weight of ideal time and cost and quality for order $o$ according to customer Wo importance weight of order $\mathrm{o}$ according to the previous step

$f_{\text {io }}$ related to raw material/component i of order o

$K^{f i o}{ }_{s}$ the largest portion of total workload of related link which could be outsourced

$I(o)$ set of raw materials/ components of order $o$

$S(i)$ set of suppliers that can supply raw materials/ components $i$

Decision Variables:

$O_{s}^{\text {fio }}$ amount of raw materials/ components $i$ of order $o$ supplied by supplier $s$

$V_{s}^{\text {fio }}$ amount of workload of $O_{s}^{f i o}$ outsourced by supplier $s$

$T T_{o}, T C_{o}, T Q_{o}$ total time, total cost, and total relative quality score

$U_{s}^{f i o}=\left\{\begin{array}{l}1 \text { if suppliers s delivers item i of order o to company } \\ 0 \text { otherwise }\end{array}\right.$

$d^{+}{ }_{o T}, d_{o T}, d^{+}{ }_{O C}, d_{O C}, d^{+}{ }_{o Q}, d_{O Q}$ positive and negative deviations from ideal time, cost and quality values for order $o$

$f_{o}$ the maximum amount of undesired deviations from ideal values for order $o$

\section{Objective Function:}

$$
\begin{aligned}
& \min z=\sum_{o} s_{o} * f_{o} \\
& T T_{o}=E T_{o}+\max _{i \in I(o)}\left\{\max _{s \in S(i)}\left\{\begin{array}{l}
P T_{i s} * O_{s}^{f_{i o}} \\
-V_{s}^{f_{i o}} \\
+D T_{s 0} * U_{s}^{f_{i o}}
\end{array}\right\}\right), \\
& \forall o=1,2, \ldots, m \\
& T T_{o} \geq P T_{i s} * O_{s}^{f_{i o}}-V_{s}^{f_{i o}}+D T_{s 0} * U_{s}^{f_{i o}}+E T_{o},
\end{aligned}
$$




$$
\begin{aligned}
& \forall o=1,2, . ., m, I(o),, s \in S\left(i_{o}\right) \\
& \frac{T T_{o}}{T G_{o}}=1+d_{o T}^{+}-d_{o T}^{-}, \quad \forall o=1,2, \ldots, m \\
& T C_{0}=E C_{o}+\sum_{i \in I(o)} \sum_{s \in S\left(i_{o}\right)}\left[\begin{array}{l}
P C i s * O_{s}^{f_{i o}} \\
+O C_{i s} * V_{s}^{f_{i o}} \\
+D C_{i s} * O_{s}^{f_{i o}}
\end{array}\right], \\
& \forall o=1,2, \ldots, m \\
& \frac{T C_{o}}{C G_{o}}=1+d_{o C}^{+}-d_{o C}^{-} \quad \forall o=1,2, \ldots, m \\
& T Q_{o}=\sum_{i \in I(o)} \sum_{s \in S\left(i_{o}\right)} \frac{Q_{i s}}{Q G_{o}} * U_{s}^{f_{i o}}, \\
& \forall o=1,2, \ldots, m \\
& T Q_{o}-\sum_{i \in I(o)} \sum_{s \in S\left(I_{o}\right)} U_{s}^{f_{i o}}=d_{o Q}^{+}-d_{o Q}^{-}, \\
& \forall o=1,2, \ldots, m \\
& f_{o} \geq a_{o T} * d_{o T}^{+} \\
& \forall o=1,2, \ldots, m \\
& f_{o} \geq a_{o C} * d_{o C}^{+} \\
& \forall o=1,2, \ldots, m \\
& f_{o} \geq a_{o Q} * d_{o Q}^{-} \\
& \forall o=1,2, \ldots, m(31) \\
& \sum_{s \in S\left(i_{o}\right)} O_{s}^{f_{i o}}=D_{o}^{*} r_{i o} \quad \forall o=1,2, \ldots, m, I(o)(32) \\
& \sum_{o} O_{s}^{f_{i 0} *} T P_{s}^{f_{i o}} \leq c_{s}+\sum_{o} V_{s}^{f_{i 0}}, \\
& \forall I(o), s \in S(i) \\
& O_{s}^{f_{i o}} \geq l_{s} * u_{s}^{f_{i o}} \\
& \forall o=1,2, . ., m, I(o), s \in S(i)
\end{aligned}
$$

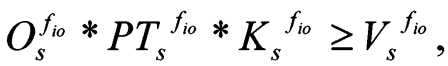

$$
\begin{aligned}
& \forall o=1,2, . ., m, I(o), s \in S(i) \\
& O_{s}^{f_{i o}} \leq M * u_{s}^{f_{i o}} \text {, } \\
& \forall o=1,2, . ., m, I(o), s \in S(i) \\
& u_{s}^{f_{i o}}=0,1 \quad \forall o=1,2, . ., m, I(o), s \in S(i)
\end{aligned}
$$

The objective function minimizes the weighted sum of maximum amount of undesired deviations from ideal values for all accepted orders. The nonlinear equation 22 computes total time of receiving all required components/materials. It is assumed that outsourced workload is done in parallel with what is done by the supplier and so it is subtracted from total time. Equation 23 is the linear form of equation 22. Total cost of receiving each order's requirements is comprised of outsourcing, manufacturing, transportation and engineering costs and is computed in equation 25. Relative quality scores of active links are computed in equations 27. Equations 24, 26 and 28 compute deviations of actual total of receiving each order's requirements from ideal values. Equations 29, 30, and 31 compute the largest undesired deviations for each order.

Equation 32 addresses supplying each order's required items. Equations 33 present capacity limitation for each supplier and the equation 34 addresses the minimum acceptable batch size for each delivered item from each supplier. The upper bound on each batch's amount of outsourced workload appears in equations 35 . Equations 36 and 37 present integrality and sign constraints, respectively.

The model has got $(3 \mathrm{mpn}+10 \mathrm{~m})$ variables with $(\mathrm{mnp})$ integer variables. The number o constraints are approximately equal to $(4 m p n+8 m+p m+p n)$. Small and medium sized problems could be solve with commercial matehmatical software like LINGO within reasonable time.

\section{CONCLUDING REMARKS}

We presented a two phased decision making framework for ranking accepted orders and supplier selection. In the first phase, customers' places in the enterprise's Customer Base as well as orders' attributes were fed into a two steps ranking procedure. A fuzzy DEA was run in the first step to approximate orders' fuzzy efficiencies. Further, a fuzzy TOPSIS was run in order to rank the orders based on the previous step's output and other introduced criteria. In the next phase, a goal programming approach was used in the supplier selection model to perform the trade-offs between conflicting objectives of cost, time and quality. taking into account the outsource of workloads to subcontractors, different combinations of time and cost were considered for each supplier and an upper bound was set to the amount of outsourced workload to increase the flexibility of the model and facilitating the trade off between cost and time. Orders' ranks computed in the first phase were fed as inputs to the model in order to ease the process of allocating limited resources in a way that high important orders receive more attention in the goals seeking process. The model outputs determine the selected supply chain and the deviations from goal values. Any deviation from the company goals of quality, cost and delivery time for the purchasing department could reveal the potential area of improvement and probable required revisions in the negotiations or contracts.

\section{NUMERICAL EXAMPLE}

To show the applicability of the model, a numerical example is presented here. An example of 5 orders with 10 components/materials in a supply chain of 10 candidate suppliers is considered. LINGO 8. Software was used to solve DEA models as well as supplier selection model. Table 1 shows the outputs of fuzzy 
DEA for computing orders' fuzzy efficiencies. the outputs are fuzzy DEA were further fed into the fuzzy TOPIS to rank the orders. Table 2 shows the results of the fuzzy TOPSIS. Selected suppliers and the supplying amounts as well as the amounts of workload to be outsourced for two sample orders are displayed in the table 3. Undesired deviations from ideal values for orders are shown in the table 4.

\begin{tabular}{c|ccc}
\multirow{2}{*}{} & \multicolumn{3}{|c}{ Table 1. Fuzzy DEA results } \\
\cline { 2 - 4 } Efficiencies \\
\cline { 2 - 4 } & 1 & $\mathrm{~m}$ & $\mathrm{u}$ \\
\hline 1 & 0.347222 & 0.604938 & 1 \\
2 & 0.3 & 0.54 & 1 \\
3 & 0.240385 & 0.495283 & 1 \\
4 & 0.171569 & 0.381264 & 0.777778 \\
5 & 0.254503 & 0.488722 & 1
\end{tabular}

Table2. Fuzzy TOPSIS results

\begin{tabular}{c|cccc} 
Orders & $\mathrm{S}_{\mathrm{i}}^{+}$ & $\mathrm{S}_{\mathrm{i}}{ }^{-}$ & $\mathrm{C}_{\mathrm{i}}$ & weight $_{\mathrm{i}}$ \\
\hline 1 & 0.346 & 0.62 & 0.641822 & 0.26552 \\
2 & 0.39 & 0.448 & 0.534606 & 0.22116 \\
3 & 0.452 & 0.49 & 0.52017 & 0.21519 \\
4 & 0.562 & 0.276 & 0.329356 & 0.13625 \\
5 & 0.476 & 0.306 & 0.391304 & 0.16188
\end{tabular}

Table 3. Supplier selection for order 2

\begin{tabular}{c|cc}
\multirow{2}{*}{ Supplier } & \multicolumn{2}{|c}{ Order 2 } \\
\cline { 2 - 3 } & produced-outsourced & $\begin{array}{c}\text { item } \\
\text { number }\end{array}$ \\
\hline & 600 & 3 \\
1 & 200 & 4 \\
& $219-2.42$ & 5 \\
\hline 2 & 400 & 7 \\
\hline 3 & 154 & 5 \\
\hline 7 & 200 & 6 \\
\hline
\end{tabular}

Table 4. Undesired deviations

\begin{tabular}{c|ccccc}
\multirow{5}{*}{\begin{tabular}{c} 
Table 4. Undesired deviations \\
Undesired \\
\cline { 2 - 6 } deviations
\end{tabular}} & 1 & 2 & 3 & 4 & 5 \\
\hline $\begin{array}{c}\mathrm{d}^{+}{ }_{\text {or }} \\
(\text { days })\end{array}$ & 0 & 1.91 & 4.09 & 0 & 6.25 \\
$\mathrm{~d}^{+}{ }_{\mathrm{OC}}(\$)$ & 0 & 56643 & 128487 & 0 & 535594 \\
$\mathrm{~d}_{\mathrm{OQQ}}(\%)$ & 0 & 0 & 0.032 & 0 & 0.088
\end{tabular}

\section{REFERENCES}

[1] Hsu, H.M., Wang, W.P., "Dynamic programming for delayed product differentiation". European Journal of Operational Research, Vol. 156, pp183-193, 2004

[2] Charnes, A., Cooper, W.W., Rhodes, E., "Measuring the efficiency of decision making units". European Journal of Operational Research, Vol. 2, pp429-444, 1978

[3] Sengupta, J.K., "A fuzzy systems approach in data envelopment analysis". Computers and Mathematics with Applications, Vol. 24, pp259-266, 1992

[4] Triantis, K., Girod, O., "A mathematical programming approach for measuring technical efficiency in a fuzzy environment". Journal of Productivity Analysis, Vol. 10, pp85-102, 1998

[5] Guo, P., Tanaka, H., "Fuzzy DEA: A perceptual evaluation method". Fuzzy Sets and Systems, Vol. 119, pp149-160, 2001

[6] Wang, Y.M., Luob, Y., Liang, L., "Fuzzy data envelopment analysis based upon fuzzy arithmetic with an application to performance assessment of manufacturing enterprises", Expert Systems with Applications, Vol. 36, pp5205-5211, 2009

[7] Hwang, C.L., Yoon, K., "Multiple attributes decision-making methods and applications", Springer, Berlin, 1981

[8] Zadeh, LA., "Fuzzy sets". Information Control, Vol. 8, pp338-353, 1965

[9] Wang Y.M., Elhag T.M.S., "Fuzzy TOPSIS method based on alpha level sets with an application to bridge risk assessment", Expert System with Application, Vol. 31, pp309-319, 2006

[10] Ghodsypour, S.H., O'Brien, C., "The total cost of logistics in supplier selection under conditions of multiple sourcing, multiple criteria and capacity constraint", International Journal of Production Economics, Vol. 3, pp15-27, 2001

[11] Cakravastia, A., Nakamura, N., "Model for negotiation the price and due date for a single order with multiple suppliers in a make-to-order environment", International Journal of Production Research, Vol. 40, pp3452-3440, 2002

[12] Liao, Z., Kuhn, A., "Operational integration of supplier selection and procurement lot sizing in supply chain", Proceedings of global project and manufacturing management symposium, Siegen Germany, 2004

[13] Ebadian, M., Rabbani, M., Jolai, F., Torabi, S.A., Tavakkoli-Moghaddam, R., "A new decision-making structure for the order entry stage in make-to-order environments", International Journal of Production Economics, Vol, 111, pp351-367, 2008

[14] Yang, Z.L., Bonsall, S., Wang, J., "Use of hybrid multiple uncertain attribute decision making techniques", Expert Systems with Applications, Vol. 36, pp1569-1586, 2009

[15] Butler, J.C., Dyer, J.S., Jia, J., Tomak, K., "Enabling e-transactions with multi-attribute preference models", European Journal of Operational Research, Vol. 186, pp748-765, 2008

[16] Rilett, L.R., Park, D., "Incorporating uncertainty and multiple objectives in real-time route selection". Journal of Transportation Engineering, Vol. 127, No. 6 , pp531-539,2001

[17] Zimmermann, H.J., "Fuzzy set theory and its application". Norwell, MA: Kluwer, 1991 